\title{
Isolated cervical lymph node metastasis of renal pelvic squamous cell carcinoma: A case report
}

\author{
Jumpei Nakadai, MD; Hiroki Ide, MD; Yosuke Hirasawa, MD; Yujiro Ito, MD; Yasumitsu Uchida, MD; \\ Takeshi Masuda, MD
}

Department of Urology, Saitama Municipal Hospital, Midoriku, Saitama, Japan

Cite as: Can Urol Assoc J 2013;7(5-6):e344-6. http://dx.doi.org/10.5489/cuaj.988 Published online May 13, 2013.

\section{Abstract}

A 78-year-old man was admitted to the Department of Otolaryngology of our hospital with bilateral lymph node swelling of the neck. Pathological examination revealed squamous cell carcinoma (SCC). He underwent computed tomography (CT) of the neck and chest, upper gastrointestinal endoscopy and laryngoscopy to locate a primary tumour, however, no obvious tumour was detected. Eight months later, a renal tumour without regional lymph node swelling was found when a chest CT scan was performed again. We then performed a right nephroureterectomy and regional lymphadenectomy. Pathological examination revealed SCC of the renal pelvis, pT3, grade 3, without regional lymph node metastasis. This pathological finding for the kidney was virtually the same as that for cervical lymph nodes. Therefore, it was thought that his cervical tumours had metastasized from the renal pelvic SCC. To the best of our knowledge, there are no reports of renal pelvic carcinoma without regional lymph node metastasis having only cervical lymph node metastasis. This is the first case of isolated cervical lymph node metastasis from renal pelvic SCC.

\section{Introduction}

Squamous cell carcinoma (SCC) of the renal pelvis is rare; it occurs in less than $10 \%$ of all patients with upper urinary tract malignancies. ${ }^{1,2}$ In almost $94 \%$ of cases, SCC of the renal pelvis, which is characterized by poor symptoms in early stages due to its tendency to invade the kidney parenchyma, is initially diagnosed as progressive stage (pT3 or higher). Therefore, SCC of the renal pelvis often metastasizes around the body when it is initially diagnosed., ${ }^{3,4}$

In patients with a renal pelvic tumour, the common metastatic sites are the regional lymph node, lung, liver and bone, while metastasis of cervical lymph nodes is uncommon. ${ }^{5}$ Generally, most cases of cervical lymph node metastasis from renal pelvic carcinoma involve regional lymph node metastasis. However, there was no regional lymph node metastasis in this case even though the patient had bilateral cervical lymph node metastasis from a renal pelvic SCC.

To our knowledge, there have been no previous reports of renal pelvic carcinoma without regional lymph node metastasis having only cervical lymph node metastasis. This is the first case of a renal pelvic SCC with isolated metastasis to cervical lymph nodes.

\section{Case report}

In July 2010, a 78-year-old man with bilateral lymph node swelling of the neck visited the otolaryngologist at our hospital. Both cytopathological and histological examinations revealed SCC from his cervical lymph nodes (Fig. 1, part a). However, a computed tomography (CT) scan of the neck and chest showed no obvious tumour in the esophagus, pharynges, larynx or elsewhere (Fig. 2, part a). Upper gastrointestinal endoscopy and laryngoscopy indicated no malignancy. The pharynges and larynx were biopsied, and the histopathological findings were negative.

In March 2011, we found a tumour in the right kidney incidentally during a chest CT scan. Its diameter was about $48 \mathrm{~mm}$, and it contracted slightly without regional lymph node swelling (Fig. 2, part b). A right nephroureterectomy and regional lymphadenectomy were performed. The pathological findings revealed SCC of the renal pelvis, pT3, grade 3 , and no metastasis to regional lymph nodes. This pathological finding was virtually the same as that of the cervical lymph nodes (Fig. 1, part b). Although upper gastrointestinal endoscopy and laryngoscopy were performed again after surgery, no obvious tumour was detected. It was thought that the cervical tumours were metastatic tumours from the renal pelvic SCC. We scheduled chemotherapy with a platinumbased combination regimen; however, the patient refused it. His general condition gradually worsened and he died in October 2011. 


\section{Discussion}

Less than $10 \%$ of all patients with upper urinary tract malignancies experience SCC of the renal pelvis. ${ }^{1,2}$ Its poor prognosis is reported to be associated with SCC of the renal pelvis due to an initial diagnosis of pT3 or higher in about $94 \%$ of cases with renal pelvic SCC. Furthermore, metastatic spread in SCC of the renal pelvis is more common than urothelial carcinoma (UC) of the renal pelvis. Therefore, the prognosis of renal pelvic SCC is very poor, with a median survival of only 7 months after surgery compared to 50 months for UC..$^{3,4}$

In patients with renal pelvic carcinoma, common metastatic sites are regional lymph nodes, lung, liver and bone, accounting for $26.3 \%, 15.8 \%, 7.0 \%$, and $3.5 \%$, respectively. Cervical lymph node metastasis is uncommon. ${ }^{5}$ Generally, most cases of renal pelvic carcinoma with cervical lymph node metastasis have regional lymph node metastasis because cancer cells spread via the lymphatic ducts from the primary organ.

However, the present case had only cervical lymph node metastasis. Although CT scan of the neck and chest, upper gastrointestinal endoscopy and laryngoscopy were performed twice in our case, they showed only a renal tumour. Therefore, it is thought that this is the case of a renal pelvic SCC with isolated metastasis to cervical lymph nodes.

To our knowledge, there have been no reports of renal pelvic carcinoma without regional lymph node metastasis having only cervical lymph node metastasis. There were two previous case reports of solitary cervical node metastasis from renal cell carcinoma (RCC). ${ }^{6,7}$ In our case, it was thought that the cancer cells metastasized only to cervical lymph nodes via the same mechanism through a thoracic duct (similar to RCC). In some cases, RCC and renal pelvic carcinoma were detected from Virchow's node metastasis as an initial finding. We believe that the metastasis in our case was spread through a thoracic duct; however, this is difficult to determine because there was no other lymph node metastasis at the same time.

The present findings indicate that the possibility of renal carcinoma should be kept in mind when an obvious primary tumour is not detected in the esophagus, pharynges, larynx or lung in patients with only cervical lymph node SCC.

\section{Conclusion}

We encountered a case of renal pelvic SCC having metastasized to cervical lymph nodes without regional lymph node metastasis. To our knowledge, this is the first case of isolated cervical lymph node metastasis from renal pelvic SCC.

Competing interests: None declared.

This paper has been peer-reviewed.

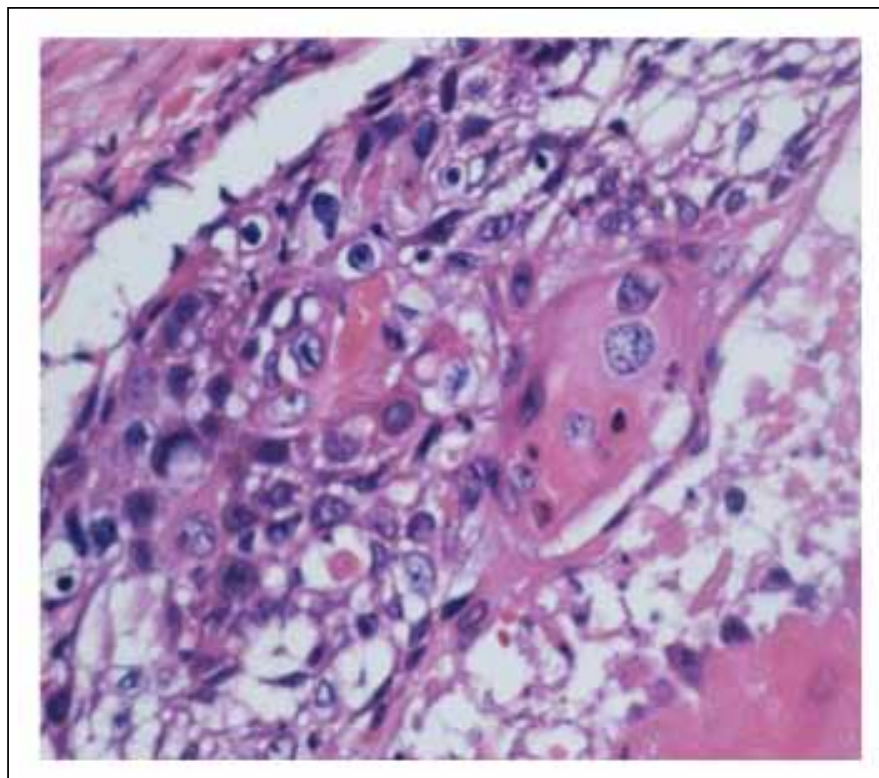

(a)

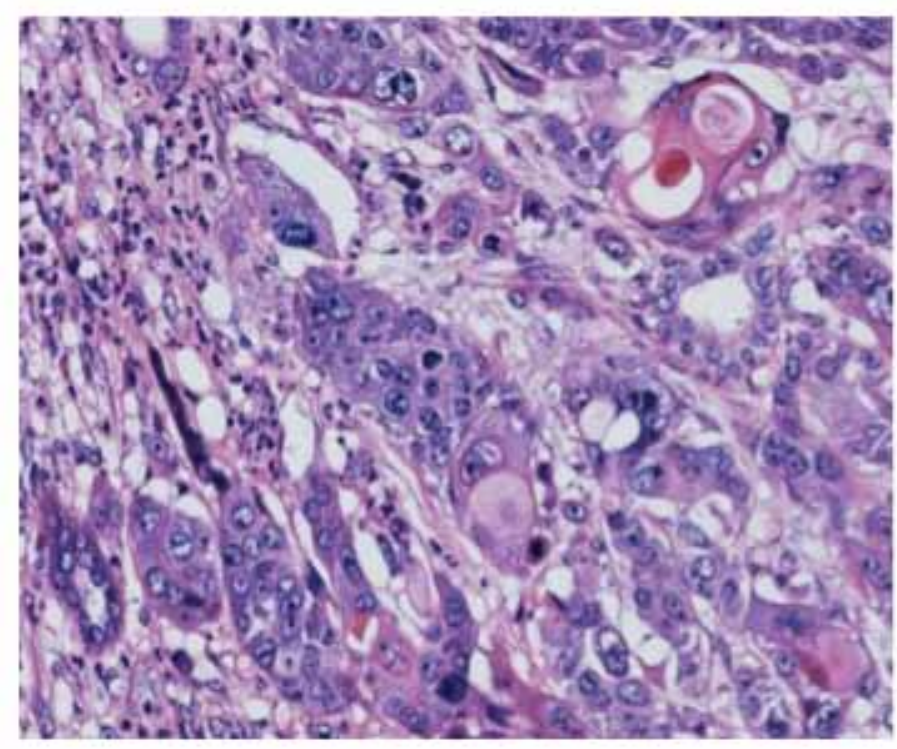

(b)

Fig 1. (a) Pathological examination of cervical lymph nodes revealed squamous cell carcinoma. (b) The pathological findings for the renal tumour were almost the same as those for the cervical lymph nodes. 
Nakadai et al.

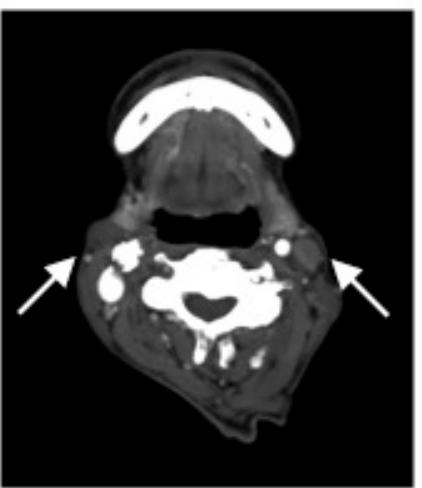

(a)

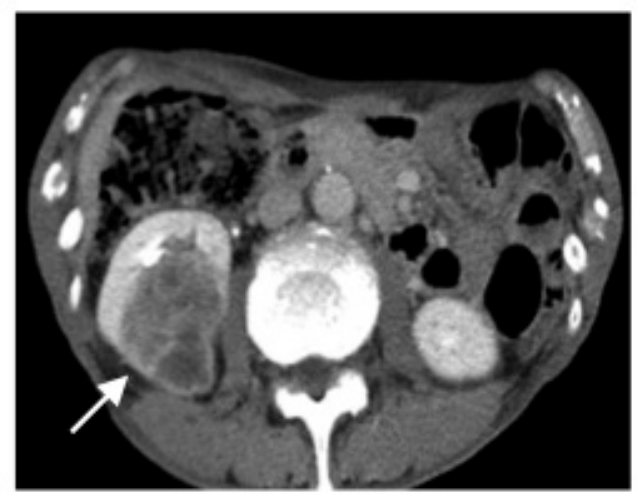

(b)

Fig 2. (a) The bilateral cervical lymph nodes were swollen, and no obvious tumour in the esophagus, pharynges or larynx was observed. (b) An abdominal computed tomography showed a tumour in the right kidney measuring $4.8 \mathrm{~cm}$ in diameter, without metastasis to regional lymph nodes.

\section{References}

1. Fukuhara H, Tamura K, Yamasaki I, et al. Granulocyte-colony stimulating factor producing squamous cell carcinoma of the renal pelvis: a case report [in Japanese]. Hinyokika Kiyo 2010;56:505-8.

2. Fujii H, Nakamura T, Mikami K, et al. Squamous cell carcinoma of the renal pelvis with elevation of G-CSF in the serum: a case report [in Japanese]. Hinyokika Kiyo 2008;54:733-6.

3. Holmang S, Lele SM, Johansson SL. Squamous cell carcinoma of the renal pelvis and ureter: incidence, symptoms, treatment and outcome. J Urol 2007;178:51-6. http://dx.doi.org/10.1016/i. juro.2007.03.033

4. Bhandari A, Alassi 0, Rogers C, et al. Squamous cell carcinoma of the renal pelvis. J Urol 2010;183:20234. http://dx.doi.org/10.1016/i.juro.2010.02.2370
5. Sengiku A, Nishiyama H, Shimizu T, et al. Post-operative recurrence patterns of urothelial tumors in the upper urinary tract: comparison between renal pelvic and ureteral tumors [in Japanese]. Hinyokika Kiyo 2008; $54: 703-9$.

6. Tzanakakis GN, McCully KS, Vezeridis MP. Solitary cervical node metastasis in renal cell carcinoma. $R$ I Med J 1990;73:113-5.

7. Savas MC, Celik I, Benekli $M$, et al. Renal cell carcinoma presenting as solitary cervical node metastasis compressing the brachial plexus. Nephron 1998;79:107-8. http://dx.doi.org/10.1159/000045003

Correspondence: Dr. Hiroki Ide, Department of Urology, Saitama Municipal Hospital, 2460 Mimuro, Midoriku, Saitama 336-8522, Japan; h-ide@fc4.so-net.ne.jp 